\title{
ESTADO NUTRICIONAL EN ESCOLARES DE PRIMERO A SEXTO GRADO EN LA PAZ, HONDURAS.
}

\author{
Nutritional status in schoolchildren from first to sixth grade in La Paz Honduras.
}

\begin{abstract}
*Allan Fernando Delcid Morazán, *Luis Enrique Delcid Morazán, *Moran E Barcan Batchvaroff, *Fabricio Alfonso Leiva Molina, ${ }^{* *}$ Delmy Soraya Barahona Andrade.
\end{abstract}

\section{RESUMEN}

Un estado nutricional deficiente en la niñez incrementa el riesgo de muerte, inhibe su desarrollo cognitivo y afecta su estado de salud de por vida. Atender esta condición es indispensable para asegurar el desarrollo de la niñez y garantizar el desarrollo del país. Objetivo: Establecer el estado nutricional en niños escolares de seis a doce años que asisten a la escuela "Nuestra Señora la Merced", La Paz, Honduras, durante el período de marzo - abril de 2016. Metodología: Estudio descriptivo de corte transversal, universo de 155 niños de primero a sexto grado, muestra 110 niños, índice de confianza 95\%, probabilidad a favor $50 \%$ y porcentaje de error $5 \%$. Previo consentimiento informado autorizado por tutores legales. Se utilizaron las tablas y guías de la OMS para valorar el estado nutricional de los niños. Resultados: De 110 estudiantes evaluados: 11 (10\%) estaban emaciados, 6 (5\%) obesos, 14 (13\%) sobrepeso, 21 (19\%) posible riesgo de sobrepeso, 58 (53\%) tenían un adecuado estado nutricional. De los niños emaciados; $40 \%$ habían padecido enfermedades respiratorias, $30 \%$ chikungunya, $10 \%$ diarrea y $10 \%$ anemia. En todos los hogares de los niños emaciados los padres reciben un ingreso mensual menor al salario mínimo. Conclusiones: Los trastornos nutricionales siguen presentes en la niñez hondureña, con un aumento significativo de niños en posible riesgo de sobrepeso y obesidad, siendo estos los principales problemas en la actualidad de los escolares en nuestro estudio, sin embargo, todavía existen niños con emaciación.

PALABRAS CLAVE: Desarrollo infantil, Desnutrición, Obesidad.

\footnotetext{
ABSTRACT

A deficient nutritional state in childhood increases the risk of death, inhibits their cognitive development and affects their health condition for life. Attending to this problem is an indispensable requirement for ensuring the development of children and the development of countries. Objective: To establish

*Doctor en Medicina y Cirugía, Universidad Nacional Autónoma de Honduras (UNAH)

${ }^{*}$ Estudiante de octavo año de Medicina y cirugía, Medico en Servicio Social, UNAH.

Dirigir correspondencia a: allanferdelcid@hotmail.com

Recibido: 28 de febrero $2017 \quad$ Aprobado: 03 de mayo 2017
}

a nutritional status in school children aged six to twelve who attend the school "Nuestra Señora La Merced", La Paz, Honduras, during the months of March through April 2016. Methology: Descriptive, cross-sectional study, with a universe of 155 children, the sample was of 110 children confidence index of $95 \%$, probability in favor $50 \%$, With a percentage error of $5 \%$, with prior informed consent authorized respectively by legal tutors, WHO tables and guidelines were used to assess the nutritional conditions of children. Results: Out of the 110 students evaluated from first to sixth grade, $11(10 \%)$ were emaciated, $6(5 \%)$ were obese, $14(13 \%)$ were overweight, $21(19 \%)$ were in risk of overweight and 58 (53\%) had an adequate nutritional condition, as well, $40 \%$ had respiratory diseases, $30 \%$ chikungunya, $10 \%$ diarrhea, and $10 \%$ anemia. In all the homes of the emaciated children parents receive a monthly income higher than the minimum salary. Conclusions: Nutritional disorders are still present in Honduran children, with a significant increase of children in possible risk of being overweight, overweight and obese, these being the main problems currently of the students in our study; however, there are still children with emaciation.

KEYWORDS: Child Development, Malnourishment, and Obesity.

\section{INTRODUCCIÓN}

La Organización Mundial de la Salud (OMS) define la desnutrición como una condición patológica la cual forma parte, junto con la sobrenutrición, de un grupo de trastornos alimentarios llamado malnutrición; las cuales son sistémicas, inespecíficas y con potencial reversible, producto de la mala utilización metabólica de los nutrientes por parte del organismo. Estas enfermedades se presentan principalmente en la niñez, sin embargo, sus complicaciones se reflejan de forma mediata y a largo plazo en el desarrollo integral del individuo. ${ }^{(1,2)}$ 
A su vez se define desnutrición como, la ingesta o absorción insuficiente de energía, proteínas o micronutrientes, que a su vez causa una deficiencia nutricional y la emaciación se define técnicamente como un valor inferior a menos dos desviaciones estándar del peso promedio para la estatura de una población de referencia. Por otro lado, la OMS define el sobrepeso y la obesidad como una acumulación anormal o excesiva de grasa que puede ser perjudicial para la salud, técnicamente el sobrepeso es el peso para la estatura con más de dos desviaciones típicas por encima de la mediana establecida en los patrones de crecimiento infantil de la OMS; y la obesidad es el peso para la estatura con más de tres desviaciones típicas por encima de la mediana establecida en los patrones de crecimiento infantil de la OMS. ${ }^{(3)}$

La desnutrición es un problema de salud pública a nivel mundial que refleja los problemas económicos y de salud; en particular, la distribución de los recursos de las naciones, se estima que existen 840 millones de niños con desnutrición en el mundo, a su vez se calcula que el $26,7 \%$ de los escolares de los países en vías de desarrollo tienen un peso insuficiente y el $32,5 \%$ no crece adecuadamente. (4-6)

En América Latina la desnutrición afecta a $88 \mathrm{mi}-$ llones de niños y refleja la falta de alimentación y nutrición adecuadas durante los años más críticos del desarrollo de los niños, la situación es particularmente grave en los países centroamericanos y Andinos. Guatemala presenta la cifra más alta de desnutrición en América Latina y El Caribe con el $46,4 \%$, seguido de Honduras con el $26,2 \%$. (7)

La nutrición está íntimamente ligada con el fenómeno biológico del crecimiento, que puede manifestarse por el aumento (balance positivo), mantenimiento (balance neutro) o disminución (balance negativo) de la masa y del volumen, que conforman al organismo, así como por la adecuación a las necesidades del cambio de forma, función y composición corporal. Un aporte inadecuado del requerimiento energético diario puede llevar a los pacientes a malnutrición. ${ }^{(8,9)}$

A su vez la nutrición tiene importancia trascendental durante los primeros años de la vida, la desnutrición favorece a las infecciones, disminuye la resistencia a casi todas las enfermedades y existe relación descrita entre el estado nutricional y las infecciones, por ende, la desnutrición actúa como causa coadyuvante de morbilidad y mortalidad infantil. La desnutrición infantil tiene una serie de consecuencias negativas en distintos ámbitos. Entre ellas destacan los impactos en educación y productividad, constituyéndose en uno de los principales mecanismos de transmisión intergeneracional de la pobreza y la desigualdad. ${ }^{(10,11)}$

Honduras presenta desnutrición infantil y 240 mil niños presentan cuadros de desnutrición por escasa ingesta de alimentos para un total de $30 \%$ de población afectada, en el país se implementó el programa de Merienda Escolar; para el año 2009 se han beneficiado 1, 322,809 con la merienda escolar, pero los componentes de la merienda se basan en maíz, arroz, frijoles y aceites, dicha dieta carece del valor nutricional adecuado para el desarrollo apropiado tanto neurocognitivo como en talla. ${ }^{(12)}$

Por lo tanto, el Objetivo de la investigación fue establecer el estado nutricional en niños escolares de seis a doce años que asisten a la escuela "Nuestra Señora La Merced", La Paz, Honduras, en los meses de marzo y abril de 2016.

\section{PACIENTES Y MÉTODOS}

Estudio de enfoque cuantitativo, alcance descriptivo y diseño transversal. Realizado en escolares de seis a doce años que asistieron a la escuela "Nuestra Señora La Merced", La Paz, Honduras, en los meses de marzo y abril de 2016. La población en estudio la conformo un universo de 155 y se calculó una muestra de 110 niños con índice de confianza $95 \%$, probabilidad a favor $(50 \%)$ y porcentaje de error del $5 \%$. La técnica de muestreo fue probabilístico, sistemático; y el procedimiento de selección de la muestra mediante tómbola. Criterios de inclusión: Niños(as) entre seis y doce años que asisten a la escuela Nuestra Señora La Merced de la ciudad de la Paz, en el período de estudio. Criterios de exclusión: Niños(as) que padecían alguna enfermedad que predisponga a desnutrición. Recolección de datos: Se realizó mediante la aplicación de un instrumento tipo cuestionario con preguntas abiertas y cerradas. La validación del mismo se realizó con una prueba piloto. Se utilizó las tablas y guías de la OMS para valorar el estado nutricional de los niños, se realizó una evaluación del estado de salud con énfasis en determinar el estado nutricional, se obtuvieron las medidas antropométricas y se aplicaron los indicadores de crecimiento como ser: longitud/ 
talla para la edad, peso para la edad, peso para la longitud/talla, Índice de masa corporal para la edad con esto se procedió a clasificar el estado nutricional de acuerdo a las Puntuaciones $Z$, ya establecidas por las tablas y guías de la OMS. Ver Tabla No 1.

Tabla No. 1. Clasificación de estado nutricional según OMS.

\begin{tabular}{|c|c|c|c|c|}
\hline \multirow[b]{2}{*}{ Puntuaciones z } & \multicolumn{4}{|c|}{ Indicadores de Crecimiento } \\
\hline & $\begin{array}{l}\text { Longitud/talla } \\
\text { para la edad }\end{array}$ & $\begin{array}{c}\text { Peso para la } \\
\text { edad }\end{array}$ & $\begin{array}{l}\text { Peso para la } \\
\text { longitud/talla }\end{array}$ & $\begin{array}{l}\text { IMC para la } \\
\text { edad }\end{array}$ \\
\hline Por encima de 3 & Ver nota 1 & \multirow{3}{*}{ Ver nota 2} & Obeso & Obeso \\
\hline Por encima de 2 & & & Sobrepeso & Sobrepeso \\
\hline Por encima de 1 & & & $\begin{array}{l}\text { Posible riesgo } \\
\text { de sobrepeso } \\
\text { (Ver nota 3) }\end{array}$ & $\begin{array}{l}\text { Posible riesgo } \\
\text { de sobrepeso } \\
\text { (Ver nota 3) }\end{array}$ \\
\hline \multicolumn{5}{|l|}{0 (mediana) } \\
\hline \multicolumn{5}{|l|}{ Por debajo de -1} \\
\hline Por debajo de -2 & $\begin{array}{l}\text { Baja talla } \\
\text { (Ver nota 4) }\end{array}$ & Bajo peso & Emaciado & Emaciado \\
\hline Por debajo de -3 & $\begin{array}{l}\text { Baja talla } \\
\text { severa } \\
\text { (Ver nota 4) }\end{array}$ & $\begin{array}{l}\text { Bajo peso } \\
\text { severo } \\
\text { (Ver nota 4) }\end{array}$ & $\begin{array}{l}\text { Severamente } \\
\text { Emaciado }\end{array}$ & $\begin{array}{l}\text { Severamente } \\
\text { Emaciado }\end{array}$ \\
\hline
\end{tabular}

Fuente: tomado de Organización Mundial de la Salud. Curso de capacitación sobre la evaluación del crecimiento del niño. Ginebra, OMS, 2008. ${ }^{(13)}$

Notas:

1. Un niño en este rango es muy alto. Una estatura alta en raras ocasiones es un problema, a menos que sea un caso extremo que indique la presencia de desórdenes endocrinos como un tumor productor de hormona del crecimiento. Si usted sospecha un desorden endocrino, refiera al niño en este rango para una evaluación médica (por ejemplo, si padres con una estatura normal tienen un niño excesivamente alto para su edad).

2. Un niño cuyo peso para la edad cae en este rango puede tener un problema de crecimiento, pero esto puede evaluarse mejor con peso para la longitud/talla o IMC para la edad.

3. Un punto marcado por encima de 1 muestra un posible riesgo. Una tendencia hacia la línea de puntuación z 2 muestra un riesgo definitivo.

4. Es posible que un niño con retardo baja talla o baja talla severa desarrolle sobrepeso.

5. Esta condición es mencionada como peso muy bajo

en los módulos de capacitación de AIEPI (Atención

Integral de las Enfermedades Prevalentes de la Infancia, Capacitación en servicio, OMS, Ginebra 1997).

Análisis estadístico: la matriz e interpretación de datos se realizó con el programa Epi Info versión 7.2, el procesamiento de datos se complementó con los programas Microsoft Excel, Word, aplicando la prueba estadística de porcentaje.

Aspectos Bióeticos: Previo a la recolección de datos se obtuvo la autorización de las autoridades de la escuela, a su vez antes de proceder a la recolección de la información, se informó a cada paciente junto con sus familiares mayores de edad o tutor legal acerca de los objetivos del estudio y sobre la confidencialidad del mismo, y brindaron su consentimiento informado, se aseguró la confidencialidad de los datos de cada participante. Todos los autores están certificados en el programa: CITI Program de la Universidad de Miami, que consiste en orientar y enseñar a los investigadores sobre las buenas prácticas clínicas en ética de la investigación, con el fin de la investigación responsable y ética de cada participante. 


\section{RESULTADOS}

De los 110 niños evaluados de primer a sexto grado (Ver Tabla No. 2) El rango del Índice de masa corpose caracterizó el estado nutricional encontrando que ral (I.M.C) se encontró entre 11.3 a 28.5 con una me$11(10 \%)$ estaban en estado de emaciación, (Ver grá- dia de 17.7. La mitad de los niños emaciados llevafico No. 1) de los cuales $6 \%$ eran niñas y $4 \%$ niños. ban merienda de sus hogares a la escuela y los que $6(5 \%)$ eran obesos, $4 \%$ masculino y $1 \%$ femenino. no llevan merienda compran alimentos en la escuela.

\section{Gráfico No. 1. Estado nutricional de los niños.}

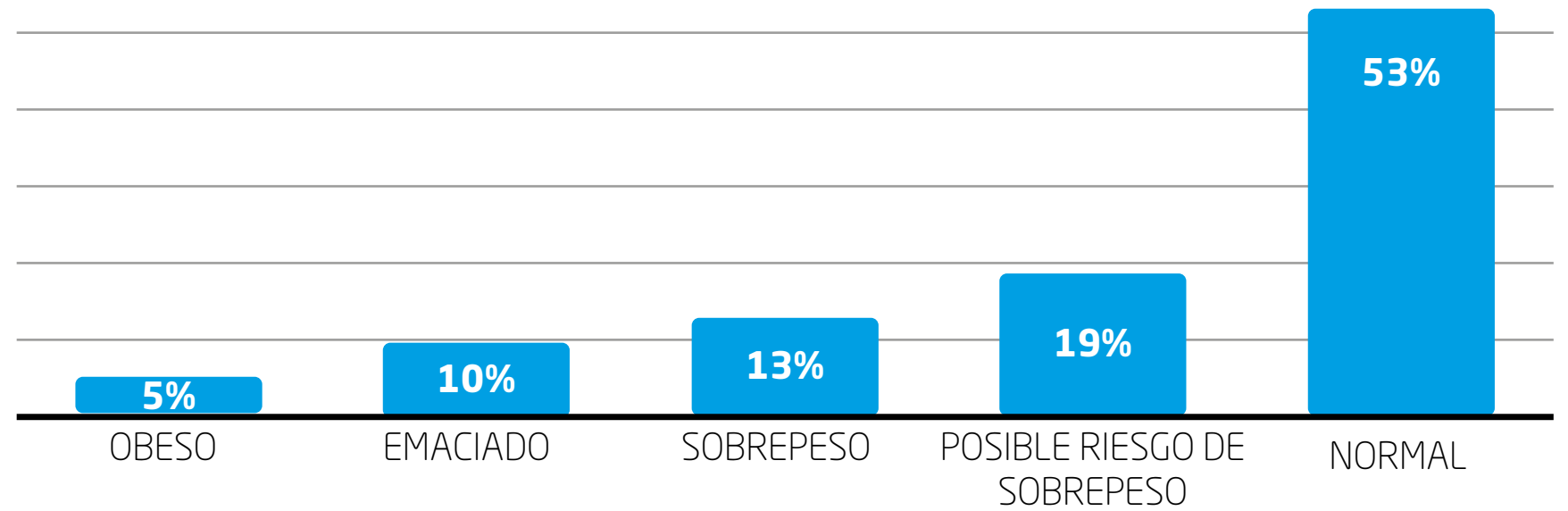

Fuente. Instrumento sobre estado nutricional en escolares de primero a sexto grado.

Tabla No. 2 Estado nutricional según el género

\begin{tabular}{|l|c|c|c|}
\hline Estado Nutricional & Masculino & Femenino & TOTAL \\
\hline Obeso & $4 \%$ & $1 \%$ & $5 \%$ \\
\hline Emaciado & $4 \%$ & $6 \%$ & $10 \%$ \\
\hline Sobrepeso & $7 \%$ & $6 \%$ & $13 \%$ \\
\hline $\begin{array}{l}\text { Posible riesgo de } \\
\text { sobrepeso }\end{array}$ & $8 \%$ & $11 \%$ & $19 \%$ \\
\hline Normal & $25 \%$ & $28 \%$ & $53 \%$ \\
\hline TOTAL & $48 \%$ & $52 \%$ & $100 \%$ \\
\hline
\end{tabular}

Fuente. Instrumento sobre estado nutricional en escolares de primero a sexto grado.

En cuanto a la distribución por grado escolar del estado nutricional se encontró que, de los niños emaciados, 4 (40\%) cursaban el quinto grado y $2(20 \%)$ segundo grado. Ver gráfico No. 2.

\section{Gráfico No. 2. Distribución de los niños emaciados} según grado escolar.

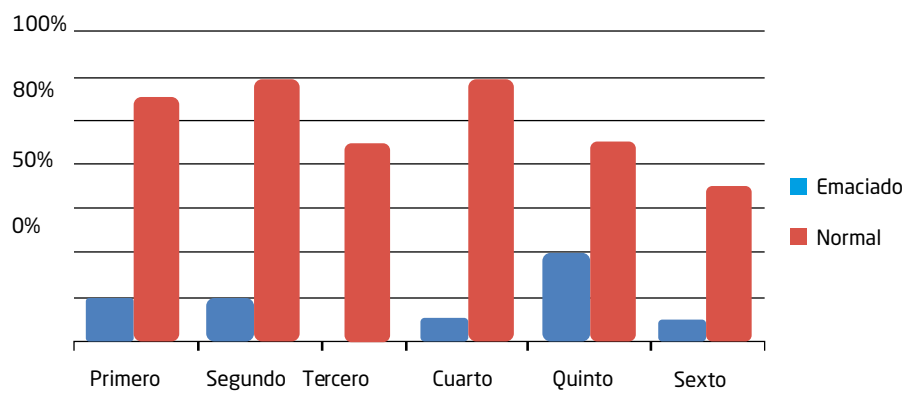

Fuente. Instrumento sobre estado nutricional en escolares de primero a sexto grado.

A su vez se encontró que el estado nutricional del 6 $(5 \%)$ de los niños obesos; 3 (50 \%) están en segundo grado, 2 (33\%) tercer grado, $1(17 \%)$ en quinto grado. En cuanto al 14 (13\%) de los niños en sobrepeso; $6(42 \%)$ se encontraban en quinto grado, $5(35 \%)$ en tercer grado, 2 (14\%) en cuarto grado, $1(9 \%)$ en segundo grado. Por último se estimó la distribución del estado nutricional por grado de los niños en posible riesgo de sobrepeso $21(19 \%)$ y se encon- 
tró que: $8(38 \%)$ se encontraban en sexto grado y 8 $(38 \%)$ en primer grado, $2(9.5 \%)$ en segundo grado, $2(9.5 \%)$ en quinto grado y $1(5 \%)$ en tercer grado.

Respecto al trabajo en las familias de los 11 (10\%) de los niños que estaban emaciados; en 6 de los hogares ningún padre trabaja y en 5 solo trabajaba un padre, a pesar de esto en todos sus hogares el ingreso mensual era menor al salario mínimo. De las 11 madres; 2 tenían secundaria incompleta, 5 secundaria completa y 4 educación superior. En 9 de los hogares existía de 3 a 4 servicios básicos mientras que 2 hogar solo contaban con 1 o 2 servicios básicos, contrario a lo que se encontró en la mayoría de los hogares con niños nutricionalmente normal 58 (53 \%) se observó que ambos padres trabajaban y sus ingresos eran mayores al salario mínimo, en todos los hogares de estos niños se cubrían los servicios básicos. En cuanto a la prevalencia de enfermedades en los $11(10 \%)$ de los niños emaciados; $40 \%$ ha padecido de enfermedades respiratorias, $30 \%$ chikungunya, $10 \%$ de diarrea, $10 \%$ anemia y $10 \%$ a padecido de otras enfermedades. De los 11 niños emaciados; 6 tenían rendimiento escolar muy bueno, 4 bueno y uno era excelente estudiante. Ninguno de los niños con desnutrición, emaciación y obesidad que formaron parte del estudio han repetido algún grado escolar, siendo el porcentaje de repitencia escolar $0 \%$. Ver tabla No. 3.

Tabla No. 3. Estado nutricional según rendimiento escolar

\begin{tabular}{|l|c|c|c|c|}
\hline Estado Nutricional & Bueno & Excelente & Muybueno & TOTAL \\
\hline Obeso & 1 & 1 & 3 & 5 \\
\hline Emaciado & 4 & 1 & 6 & 11 \\
\hline Sobrepeso & 3 & 4 & 7 & 14 \\
\hline Posible riesgo de sobrepeso & 1 & 12 & 8 & 21 \\
\hline Normal & 10 & 23 & 26 & 59 \\
\hline TOTAL & $\mathbf{1 9}$ & $\mathbf{4 1}$ & $\mathbf{5 0}$ & $\mathbf{1 1 0}$ \\
\hline
\end{tabular}

Fuente. Instrumento sobre estado nutricional en escolares de primero a sexto grado.

\section{DISCUSIÓN:}

Honduras es uno de los países con mayor índice de desnutrición en América Latina, sin embargo, se cuenta con pocos estudios realizados en el país, ninguno de ellos del departamento de La Paz. De los 110 niños encuestados se encontró que el $10 \%$ estaba emaciado y se observó que es mayor el porcentaje de niños con obesidad $5 \%$, sobrepeso $13 \%$ y en posible riesgo de sobrepeso $19 \%$, sumando en total $37 \%$, estos datos sugieren que hay mayor tendencia a los trastornos relacionados con la obesidad, dato similar a un estudio en la población escolar de Tegucigalpa, Honduras en el 2005 que presentó bajo peso en el $2 \%$ de los niños, $13 \%$ sobrepeso y $6 \%$ obesidad, ${ }^{(14)}$ a su vez concuerda con un estudio reciente que indica que la prevalencia de obesidad en dos comunidades rurales una de Cortes y otra de Santa Bárbara, Honduras es significativa, variando según el sexo y la edad, ${ }^{(15)}$ ambos datos nacionales son similares y concuerdan con estudios internacionales que indican que los trastornos relacionados con la desnutrición han disminuido pero se ha observado un aumento con los trastornos nutricionales relacionados con la tendencia al sobrepeso y obesidad. (16) Ninguno de los niños con emaciación, obesidad, sobrepeso y en posible riesgo de sobrepeso tiene mal rendimiento académico, independientemente de su 
estado nutricional, tampoco han repetido algún grado escolar, ni han abandonado la escuela, esto pudiera deberse a la metodología de educación implementada por parte del ministerio de educación y el programa "Todos podemos avanzar" que se adoptó para evitar un sistema de educación excluyente y expulsador, ${ }^{(17)}$ estos resultados son peculiares y contradictorios a lo que se reporta en la literatura internacional, ya que indica, que es imperativo mantener un adecuado estado nutricional porque el desarrollo cognitivo está vinculado con la nutrición de los niños, un estado nutricional deficiente tiene efectos adversos sobre el proceso de aprendizaje y el rendimiento escolar, asimismo los efectos de un mal estado nutricional en los primeros años se prolongan a lo largo de la vida, ya que incrementan el riesgo de padecer enfermedades crónicas (sobrepeso, obesidad, diabetes, enfermedades cardiovasculares) y esto está asociado con menores logros educativos en la adultez. $(18,19)$

A su vez un estudio sobre desnutrición realizado en una aldea de Intibucá, Honduras, reflejo que las enfermedades más frecuentes en la población son las respiratorias en $79.2 \%$ y enfermedades diarreicas en un $19 \%$, estos datos coinciden con los obtenidos en el presente estudio, ya que, de los niños con emaciación, las enfermedades más frecuentes fueron las enfermedades respiratorias 40\%, Chikungunya $30 \%$, anemia $10 \%$ y diarreas $10 \%$. Cabe recalcar que tanto en la experiencia clínica, como la experimental señalan que la desnutrición y la infección se entrelazan íntimamente potenciando sus efectos en forma recíproca. Es así como en la desnutrición aumenta la incidencia y gravedad de las infecciones y estas últimas al repetirse agravan la desnutrición, determinándose un circulo difícil de superar. ${ }^{(20,21)}$

En la mayoría de los hogares con niños nutricionalmente normal $53 \%$ se observó que ambos padres trabajaban y sus ingresos eran mayores al salario mínimo, pero en cuanto al $10 \%$ de niños emaciados se encontró que el ingreso mensual es inferior al salario mínimo, esto refleja un artículo de factores económicos relacionados con la nutrición en países en desarrollo, que indica que el ingreso en efectivo pueden incrementar el estado nutricional de los niños debido a que los padres eliminarían la restricción económica y distribuirían los recursos hacia las necesidades más apremiantes de sus hijos, comoalimentos nutritivos. ${ }^{(22)}$

En conclusión: Los trastornos nutricionales siguen presentes en la niñez hondureña, con aumento sig- nificativo de niños en posible riesgo de sobrepeso, sobrepeso y obesidad, siendo estos los principales problemas en la actualidad de los escolares que asisten a la escuela "Nuestra Señora la Merced", La Paz, Honduras, sin embargo, todavía existen niños con emaciación, esto debido principalmente al bajo ingreso económico de la población que limita la accesibilidad a una canasta básica balanceada.

Se recomienda: A la Secretaria de Salud y a la Secretaria de Educación se les sugiere la creación de un programa de alimentación escolar balanceado, con valor nutricional que no tenga efecto negativo en los niños en edad escolar, siendo este supervisado y evaluado por personal de la salud, capacitado en el tema por la Universidad Nacional Autónoma de Honduras.

\section{CONFLICTO DE INTERÉS}

Los autores declaran no tener conflictos de interés con la publicación de este artículo.

\section{REFERENCIAS BIBLIOGRAFICAS}

1. Organización Mundial de la Salud [sede web]. Ginebra: OMS; 2016. [Actualizado 2017 citado 2017 Feb. 18]. ¿Qué es la malnutrición? Disponible en: http://www.who.int/features/qa/malnutrition/es/

2. Pleus J. Alteraciones del estado nutricional. En: Mattson Porth C. Fisiopatología salud-enfermedad: un enfoque conceptual. $7^{\mathrm{a}}$ ed. Madrid: Médica panamericana; 2006. p. 216-229.

3. UNICEF. Glosario de nutrición: un recurso para comunicadores. [Internet]. UNICEF; 2012. [Actualizado 2012; citado 2017. Marzo. 25]. Disponible en: https://www. unicef.org/lac/Nutrition_Glossary_ES.pdf.

4. Ortega-Bonilla RA, Chito-Trujillo DM. Valoración del estado nutricional de la población escolar del municipio de Argelia, Colombia. Rev. salud pública. [ Revista en internet]. 2014 [Acceso el 12 de junio del 2015];16(4):547-559.Disponible en: http://www.redalyc.org/pdf/422/42235721006.pdf

5. Neufeld LM, Hernández S, Fernández AC. Desnutrición crónica en Centroamérica e inventario de intervenciones nutricionales, versión 2. Cuernavaca, Morelos: Dirección de Epidemiología de la Nutrición; 2006.

6. William C. Necesidades nutricionales. En: Klieg- 
man, Stanton BF, St. Geme JW, Behrman RE, editores. Nelson tratado de pediatría. $18^{\mathrm{a}}$ ed. Barcelona:ElsevierSaunders;2008. p. 209-2014225-227.

7. UNICEF. Desnutrición Infantil en América Latina y El Caribe Desafíos. Boletín de la infancia y adolescencia. [Internet].2006 [Citado 24 de febrero 2017];(2). Disponible en: https:// www.unicef.org/lac/Desafiosnutricion(13).pdf

8. Ortiz-Andrellucchi A, Peña Quintana L, Albino Beñacar A, Mönckeberg Barros F, Serra-Majem L. Desnutrición infantil, salud y pobreza: intervención desde un programa integral. Nutr. Hosp. 2006; 21(4):533-541.

9. Angulo L, Meza C. Diagnóstico nutricional en escolares de los municipios Libertador, Campo Elías, Santos Marquina y Sucre del estado Mérida. Educere. 2013; 17(58):515-524.

10. Cordero-Herrera AM. Principales enfermedades asociadas al estado nutricional en el niño menor de un año. Medicentro Electrón. 2014;18(3).

11. SINDIC de Greuges. Informe sobre la malnutrición infantil en Cataluña. Cataluña: SINDIC; 2013.

12. Márquez-González $H$, García-Sámano VM, Caltenco-Serran M, García-Villegas EA, Márquez-Flore $\mathrm{H}$, Villa-Romer AR. Clasificación y evaluación de la desnutrición en el paciente pediátrico. El Residente. 2012; 7(2):59-69.

13. Organización Mundial de la Salud. Curso de Capacitación sobre la evaluación del crecimiento del niño. Ginebra: OMS; 2008.

14. Rivera M. Obesidad en condiciones de pobreza, estudio epidemiológico en escolares de escuelas públicas de Tegucigalpa, Honduras. Rev. Med. Hondur 2005; 73(1):10-14.

15. Fuentes CA, Guerra JJ, Abujieras CM, et al. Prevalencia de obesidad en escolares en dos comunidades rurales. Rev. Cient. Esc. Univ. Cienc. 2016;3(1):25-29.

16. Ramirez-Pajuelo J. Abanto-Sánchez J. Dongo-Álvarez D. Mamani-Tarqui C. Zamora-Agüe- ro R. Sobrepeso, obesidad y Desnutrición Crónica en niños de 6 a 9 años en Perú 20092010. Rev Peru Med Exp Salud Publica. [ Revista en internet]. 2013 [Citado 03 de marzo 2017]; 30(4):583-9. Disponible en: http://www. scielosp.org/pdf/rpmesp/v30n4/a07v30n4.pdf

17. Más de 36,000 alumnos son repitentes este año en Honduras. [ en línea]. Honduras: La Prensa; 2015. Disponible en:http:// www.laprensa.hn/honduras/865460-410/m\%C $3 \%$ A 1 s-de 36000 alumnos-son-repitentes-este-a\%C3\%B10-en-honduras

18. Instituto Nacional de Estadísticas e Informática (INEI). Nutrición de los niños. En: Instituto Nacional de Estadísticas e Informática (INEI). Encuesta demográfica y salud familiar Perú 20072008. [en internet] Perú: INEI:2009. 226-233. [Accesada 5 de diciembre] Disponible en: https:// dhsprogram.com/pubs/pdf/FR234/FR234.pdf

19. Arzapalo-Salvador F. Pantoja-Villalobos K. Romero-López J. Farro-Peña G. Estado nutricional y rendimiento escolar de los niños de 6 a 9 años del Asentamiento Humano Villa Rica - Carabayllo Lima - Perú 2011. Rev. Enferm. Herediana 2011; 4(1):20-26.

20. Cortes MM, Buchanan JC, Vásquez M, Bobadilla $L$. La desnutrición y su impacto en la educación pre básica en la aldea, Arcilaca de Honduras. Rev. Fac. Cienc. Méd. 2007; 4(1):23-32.

21. Schlesinger L. Stekel A. Alteraciones inmunológicas en la desnutrición. Rev. chil. pediatr. [revista en internet].1973 [Acceso el 12 de mayo del 2016];44(5). Disponible en: http://www.scielo.cl/pdf/rcp/v44n5/art08.pdf.

22. Velásquez Valdivia A. Factores Económicos asociados a la nutrición e impacto de programas de reducción de la pobreza en la desnutrición de países en desarrollo. [Internet]: PREVAL;2005. [citado 15 de enero]. Disponible en: http://www.midis.gob. pe/dgsye/evaluacion/documentos/ev_Revision Sistematica_economia_nutricion_ $\bar{A} V$ _fin.pdf 